\title{
The Feasibility, Safety and Effectiveness of a Ketogenic Diet for Refractory Status Epilepticus in Adults in the Intensive Care Unit
}

\author{
Brandon A. Francis ${ }^{1 *}$, Jennifer Fillenworth², Philip Gorelick ${ }^{3}$, Kristina Karanec ${ }^{2}$ and Adriana Tanner ${ }^{2}$
}

\begin{abstract}
Background: Status, refractory status and super refractory status epilepticus are common neurologic emergencies. The objective of this study is to investigate the feasibility, safety and effectiveness of a ketogenic diet (KD) for refractory status epilepticus (RSE) in adults in the intensive care unit (ICU).

Methods: We performed a retrospective, single-center study of patients between ages 18 and 80 years with RSE treated with a KD treatment algorithm from November 2016 through April 2018. The primary outcome measure was urine ketone body production as a biomarker of feasibility. Secondary measures included resolution of RSE and KDrelated side effects.

Results: There were 11 adults who were diagnosed with RSE that were treated with the KD. The mean age was 48 years, and $45 \%(n=5)$ of the patients were women. The patients were prescribed a median of three anti-seizure medications before initiating the KD. The median duration of RSE before initiation of the KD was 1 day. Treatment delays were the result of Propofol administration. $90.9 \%(n=10)$ of patients achieved ketosis within a median of 1 day. RSE resolved in $72.7 \%(n=8)$ of patients; however, $27.3 \%(n=3)$ developed super-refractory status epilepticus. Side effects included metabolic acidosis, hypoglycemia and hyponatremia. One patient (20\%) died.
\end{abstract}

Conclusions: KD may be feasible, safe and effective for treatment of RSE in the ICU. A randomized controlled trial (RCT) may be indicated to further test the safety and efficacy of KD.

Keywords: Ketogenic diet, Neurocritical care, Refractory status epilepticus

\section{Introduction}

Status epilepticus (SE), refractory status epilepticus (RSE) and super-refractory status epilepticus (SRSE) are major causes of neurologic morbidity and mortality [1-3]. After stroke, SE is the second most common neurologic emergency accounting for approximately $1 \%$ of all emergency department visits and is associated with a mortality rate as high as $27 \%$ [2]. RSE and SRSE have mortality rates approaching $22 \%$ and up to $30-50 \%$ [3]. Rapid seizure control, however, may allow patients to return to their baseline

\footnotetext{
*Correspondence: Brandon.francis@mercyhealth.com

${ }^{1}$ Neurocritical Care, Hauenstein Neurosciences Mercy Health Saint Mary's,

200 Cherry Street, Grand Rapids, MI 49503, USA

Full list of author information is available at the end of the article
}

neurologic function [4]. The ketogenic diet (KD), a low carbohydrate diet that promotes formation of ketone bodies (e.g., acetoacetate and beta-hydroxybutyrate) based on a shift to fat metabolism, has been effective in the treatment of epilepsy and shows promise as a possible therapy for SE [5-9]. We carried out a single-center study of the administration of a KD to adult patients in an intensive care unit (ICU) who had RSE and now describe our findings.

\section{Methods}

\section{Study Design}

This is a single-center retrospective study evaluating whether adults with RSE in the ICU can achieve ketosis using a standardized KD approach. Secondary outcomes 
include resolution of seizures and documented side effects associated with the KD. The study was carried out from November 2016-May 2018.

\section{Standard Protocols and Consents}

The study was approved by the Mercy Health Saint Mary's Institutional Review Board (IRB), and since it was a retrospective data analysis, the KD was offered as a treatment option for the patient to the next-of-kin or designated power of attorney. This study adheres to the ethical standards defined by the IRB.

\section{Support}

The study did not receive any external support. The institutional study staff were supported by the Institution.

\section{Population}

The study population consisted of a convenience sample of adults with RSE who were aged 18-80 years and administered the KD as part of multimodal treatment for RSE.

\section{Definitions}

We operationally defined seizures and various types of SE in the following manner:

Seizures-abnormal, synchronous, paroxysmal neuronal discharges that cause alterations in thought, behavior or sensation [1].

Status epilepticus - a seizure or recurrent seizures lasting 5 min without return to baseline.

Refractory status epilepticus-continued or repeated seizures that do not respond to appropriate treatment for SE (e.g., benzodiazepine) and a second-line pharmacologic agent (e.g., Phenytoin).

Super-refractory status epilepticus-seizures that persist or reemerge in the setting of intravenous anesthetic infusions $\times 24 \mathrm{~h}$ used for the treatment of SE [2].

Our center follows the Salzburg Consensus Criteria for the diagnosis of non-convulsive status epilepticus. We consider the following: a minimum duration of $10 \mathrm{~s}$; burden of epileptiform discharges $>2,5 \mathrm{~Hz}$; presence of rhythmic delta/theta activity; evolution (frequency, amplitude and spatiotemporal); presence of fluctuation of epileptiform discharges and/or rhythmic delta/theta; and presence of improvement after intravenous anti-epileptic drugs [10].

Resolution of RSE was determined both clinically and electrographically (i.e., the Salzburg Consensus Criteria).

\section{KD Protocol}

The KD protocol is outlined in Fig. 1.

\section{Data Collection}

Data collection included patient demographics, determination of the presumed etiology of RSE, number of anticonvulsant drugs used prior to initiation of $\mathrm{KD}$, continuous video electroencephalography monitoring results, urine ketone results, and site of placement after ICU discharge. Also, adverse events were recorded in an unstructured approach.

\section{Outcome Variables}

The presence or absence of urinary ketones was the primary outcome measure. Secondary measures included resolution of RSE or progression to SRSE and 6-month seizure outcomes (if available), at discharge and follow-up.

\section{Descriptive Study Statistics}

Proportions were calculated for categorical variables, and means, medians and interquartile ranges (IQRs) were calculated for continuous variables as appropriate.

\section{Data Availability}

Anonymized data including the KD source and administration protocol, patient length of stay, burst suppression information and other clinical details can be made available to qualified investigators upon request to the corresponding author after appropriate review by the Saint Mary's IRB in accordance with its policies.

\section{Results}

\section{Clinical Characteristics}

Eleven patients met the study eligibility criteria. The mean age was 48 years, and $45 \%(n=5)$ were women. Seventy-three percent $(n=8)$ were Caucasian. Pretreatment diagnoses related to epilepsy are shown in Table 1. Forty-one percent $(n=5)$ had pre-morbid epilepsy. The underlying etiology of RSE (Table 1 ) included traumatic brain injury $(n=3)$, post-cardiac arrest $(n=2)$, spontaneous intracerebral hemorrhage $(n=2)$, ischemic stroke $(n=1)$, poor adherence to anticonvulsant medication $(n=1)$, alcohol withdrawal $(n=1)$ and $N$-methyl-D-aspartate receptor encephalitis $(n=1)$. The median number of anti-seizure drugs (ASDs) prior to KD initiation and total ASD requirement both totaled three drugs.

\section{Outcomes After KD Initiation}

Ten out of the eleven patients (90.9\%) achieved ketosis (i.e., generated urine ketones) with a median of 1 day. Treatment delays in initiating the KD were due to administration of Propofol $(18.1 \%, n=2)$. RSE resolved in 

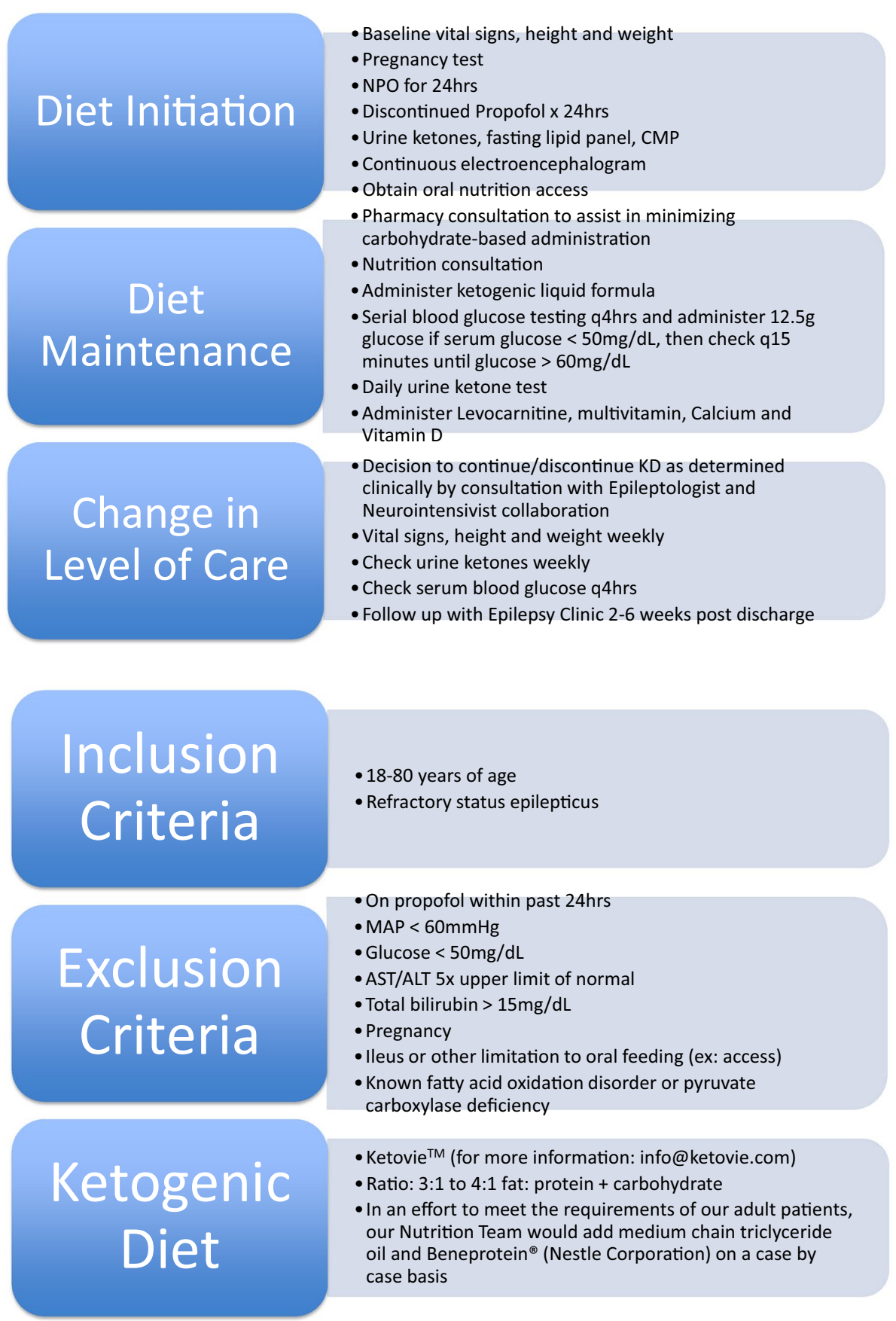

Fig. $1 \mathrm{KD}$ treatment algorithm and main study eligibility criteria

72.7\% ( $n=8)$; however, 27.3\% $(n=3)$ developed SRSE. The three underlying etiologies that progressed to SRSE were NMDA-receptor encephalitis, epilepsy and cardiac arrest. Of those with 6-month follow-up data $(n=5)$, one patient died (20\%). Of the two patients who suffered cardiac arrest, one patient survived. The other patient had a change in goals of care to reflect the patient's wishes of comfort measures only and withdrawal of life support (Table 2).

The most common side effect of the KD was metabolic acidosis $(72.2 \% ; n=8)$ followed by hypoglycemia $(18.1 \%$; $n=2)$. Of note, Patient 1 had a complicated clinical course including bowel perforation and numerous infections. The bowel perforation was believed to be related 
Table 1 Demographics

\begin{tabular}{|c|c|c|c|c|c|c|c|c|}
\hline Patient & Age (year) & Gender & Ethnicity & Diagnosis & Epilepsy & $\begin{array}{l}\text { Duration of sta- } \\
\text { tus (days) }\end{array}$ & ASD pre-KD & ASD post-KD \\
\hline 1 & 21 & Female & Caucasian & NMDA & No & 3 & 2 & 9 \\
\hline 2 & 31 & Female & Caucasian & $\mathrm{TBI}$ & Yes & 1 & 3 & 3 \\
\hline 3 & 31 & Male & AA & $\mathrm{ICH}$ & Yes & 2 & 4 & 4 \\
\hline 4 & 36 & Female & Caucasian & CA & No & 0 & 3 & 4 \\
\hline 5 & 72 & Male & Caucasian & Stroke & No & 2 & 2 & 3 \\
\hline 6 & 68 & Male & East Asian & $\mathrm{TBI}$ & Yes & 0 & 2 & 3 \\
\hline 7 & 24 & Male & Caucasian & Epilepsy & Yes & 0 & 8 & 10 \\
\hline 8 & 73 & Female & Caucasian & $\mathrm{ICH}$ & No & 3 & 2 & 2 \\
\hline 9 & 46 & Male & Caucasian & CA & No & 2 & 2 & 6 \\
\hline 10 & 52 & Male & Caucasian & TBI & Yes & 0 & 3 & 3 \\
\hline 11 & 72 & Female & AA & $\mathrm{EtOH}$ & No & 0 & 3 & 3 \\
\hline
\end{tabular}

AA African American, ASD anti-seizure drugs, CA cardiac arrest, EtOH ethanol withdrawal, KD ketogenic diet, NMDA n-methyl-d-aspartate receptor encephalitis, TBI traumatic brain injury

to the placement of a surgical feeding tube, and multiple infections were believed to be related to administration of immunosuppressive therapy.

\section{Discussion}

We report a retrospective study that demonstrates that 91\% of our patients with RSE achieved ketosis using the KD within 1 day of initiation. There was one patient (patient 11) that demonstrated urine ketones on only one laboratory determination, had a side effect of hypoglycemia, but was able to continue the KD.

Thus, in our small sample of select patients, it was feasible to administer the KD and achieve ketosis. Similar to other studies, we utilized urine ketones as our primary outcome measure to determine whether patients achieved ketosis [11]. We found that all 11 of our patients with RSE (73\%) and those who developed SRSE (27\%) ultimately had complete resolution of seizures with a combination of ASD and the KD.

Our findings add to the recent growing body of evidence that the KD is feasible and safe. A recent pediatric study evaluated the efficacy of KD for convulsive SE. The authors found that $71 \%$ of their patients demonstrated resolution of their seizures within 7 days. Similar to our observations, the authors noted ketosis was achieved at median of 2 days. Only 1 out of the 14 pediatric patients had to discontinue the KD due to gastrointestinal issues. This pediatric study differs from our data presented here in that the participants initiated the KD later at a median of 13 days [12].

Table 2 Primary outcome and safety

\begin{tabular}{|c|c|c|c|c|c|c|}
\hline Patient & ASD total & Ketosis & $\begin{array}{l}\text { Time to ketosis } \\
\text { (days) }\end{array}$ & Adverse events & ICU LOS (days) & Disposition \\
\hline 1 & 9 & Yes & 2 & MA, Abd Perf, Infxn, HypoGly & 87 & Rehab \\
\hline 2 & 3 & Yes & 5 & MA, Transaminitis & 2 & Home \\
\hline 3 & 4 & Yes & 1 & None & 5 & Home \\
\hline 4 & 4 & Yes & 1 & None & 9 & $\mathrm{CM}$ \\
\hline 5 & 3 & Yes & 1 & MA & 5 & Rehab \\
\hline 6 & 3 & Yes & 3 & HypoNa, MA & 9 & SNF \\
\hline 7 & 10 & Yes & 0 & MA & 29 & LTACH \\
\hline 8 & 2 & Yes & 2 & MA & 15 & LTACH \\
\hline 9 & 6 & Yes & 0 & MA & 23 & $\mathrm{LTACH}$ \\
\hline 10 & 3 & Yes & 0 & None & 2 & SNF \\
\hline 11 & 3 & No & $\mathrm{n} / \mathrm{a}$ & HypoGly & 19 & LTACH \\
\hline
\end{tabular}

ASD anti-seizure drugs, Abd Perf bowel perforation, $C M$ comfort measures, withdrawal of life support, HypoGly hypoglycemia (serum level < $60 \mathrm{mg} / \mathrm{dL}$ ), HypoNa hyponatremia, ICU intensive care unit, Infxn infection, LOS length of stay, LTACH long-term acute care hospital, MA metabolic acidosis (pH $<7.40 \mathrm{mmHg}$ or serum bicarbonate $<24 \mathrm{mEq} / \mathrm{L})$, Rehab rehabilitation, SNF skilled nursing facility 
In our cohort, the $\mathrm{KD}$ was relatively well tolerated with the most common side effects being metabolic acidosis and hypoglycemia. Only one of the patients had to suspend the KD due to an adverse event. Patient 1 required bowel rest after a documented bowel perforation.

The recent prospective trial by Cervenka and colleagues demonstrates the feasibility of administration of the KD to ICU patients with SRSE [9]. Our retrospective study is important as it shows the feasibility of utilizing the KD early in the treatment course for patients with RSE. Furthermore, our findings suggest the possibility of administration of the $\mathrm{KD}$ as a prophylactic to minimize the risk of developing SRSE. Such a prophylactic approach could be important as it has been demonstrated that there is an increase in mortality as the clinical course of SE progresses from RSE to SRSE. The mortality increase is likely multifactorial in causation including complications associated with difficult to treat seizures and the complications of the treatment itself (including intravenous anesthetic infusions, increased vasopressor requirements, decreased ventilator-free days) $[2,13,14]$. As seizures progress from RSE to SRSE, it is not uncommon for the number of ASDs administered to increase [9]. Our patients required a median of three ASDs which highlights another potential benefit of the KD as part of a treatment strategy for RSE-reduction in the number of ASDs needed to treat the condition.

A recent meta-analysis that included 16 randomized trials with a total of 3225 patients, evaluated the effects of early enteral nutrition in ICU patients. The authors found that there was no difference between early enteral nutrition and other forms of nutritional support with regards to mortality. However, in pre-specified subgroup analysis (that included mixed ICU populations) there was a mortality benefit (OR 0.45 ; 95\% CI, $0.21-0.95 ; p=0.038$ ) along with a decreased risk of developing pneumonia (OR 0.75; 95\% CI, 0.60-0.94; $p=0.012$ ) in patients that received early enteral nutrition (within 1-2 days of ICU admission) [15]. The aforementioned data help support our findings that an early enteral nutrition approach does not appear to be harmful and may be helpful.

Our study has several limitations including the retrospective nature of the study which may be subject to confounding and spurious findings. The patients were evaluated for inclusion based on physician preference which may limit the ability to generalize these data. Furthermore, in this cohort $27 \%$ of the patients with RSE progressed to SRSE which is slightly higher than some prior reports [16]. This may reflect the fact that our institution is a referral center for community hospitals and subsequently our patients may harbor a form of RSE that is more likely to progress to SRSE. This could also be related to the small sample size such that progression to SRSE was over represented among our patient population. In addition, we have limited follow-up data such that only $45 \%(n=5)$ of our patients were available for longer-term analysis. Of those patients, one died (20\%) and that was due to withdrawal of life support in accordance with the patient's previously stated wishes. Finally, our study was a descriptive retrospective one subject to selection bias and confounding, and ranks lower on the hierarchy of epidemiologic studies compared to a randomized controlled trial (RCT) design.

\section{Conclusion}

We present a retrospective study of 11 adult patients with RSE who successfully and relatively quickly and safely achieved ketosis utilizing the KD. This study provides preliminary data to help support a future RCT of the KD as part of a treatment regimen for RSE and prophylaxis of SRSE.

\section{Author details \\ 1 Neurocritical Care, Hauenstein Neurosciences Mercy Health Saint Mary's, 200 Cherry Street, Grand Rapids, MI 49503, USA. ${ }^{2}$ Epilepsy Center, Hauenstein Neurosciences Mercy Health Saint Mary's, Grand Rapids, MI, USA. ${ }^{3}$ Vascular Neurology, Hauenstein Neurosciences Mercy Health Saint Mary's, Grand Rapids, MI, USA.}

\section{Author Contributions}

BAF contributed to study concept and design, data acquisition, data analysis, drafting the manuscript and statistical analysis. JF was involved in ketogenic diet administration protocol. PG and KK critically reviewed, edited and revised the manuscript. AT was involved in study concept, critical review, editing and revision of the manuscript.

\section{Source of support}

This study did not receive external funding or support. Institutional study staff were supported by the institution

\section{Compliance with Ethical Standards}

\section{Conflict of interest}

Dr. Tanner has received honoraria for consulting and the Speaker's Bureau from Eisai Inc., and Sunovion Inc. Remaining authors have no conflict of interest.

\section{Ethical Approval}

The study was approved by the Mercy Health Saint Mary's Institutional Review Board (IRB), and since it was a retrospective data analysis, the KD was offered as a treatment option for the patient to the next-of-kin or designated power of attorney. This study adheres to the ethical standards defined by the IRB.

Published online: 27 November 2018

\section{References}

1. Stafstrom CE, Carmant L. Seizures and epilepsy: an overview for neuroscientists. Cold Spring Harbor perspectives in medicine, vol. 5. New York: Cold Spring Harbor Laboratory Press; 2015. p. a022426. 
2. Claassen J, Riviello JJ, Silbergleit R. Emergency neurological life support: status epilepticus. Neurocrit Care. 2015;23:136-42.

3. Kantanen A-M, Reinikainen M, Parviainen I, Ruokonen E, Ala-Peijari M, Bäcklund T, et al. Incidence and mortality of super-refractory status epilepticus in adults. Epilepsy Behav. 2015;49:131-4.

4. Brophy GM, Bell R, Claassen J, Alldredge B, Bleck TP, Glauser T, et al. Guidelines for the evaluation and management of status epilepticus. Neurocrit Care. 2012;17:3-23.

5. Gano LB, Patel M, Rho JM. Ketogenic diets, mitochondria, and neurological diseases. J Lipid Res. 2014;55:2211-28.

6. Klein P, Tyrlikova I, Mathews GC. Dietary treatment in adults with refractory epilepsy: a review. Neurology. 2014;83:1978-85.

7. Thakur KT, Probasco JC, Hocker SE, Roehl K, Henry B, Kossoff EH, et al. Ketogenic diet for adults in super-refractory status epilepticus. Neurology 2014;82:665-70.

8. Friedman D, Claassen J, Hirsch LJ. Continuous electroencephalogram monitoring in the intensive care unit. Anesth Analg. 2009;109:506-23.

9. Cervenka MC, Hocker S, Koenig M, Bar B, Henry-Barron B, Kossoff EH, et al, Phase I/II multicenter ketogenic diet study for adult superrefractory status epilepticus. Neurology. 2017;88:938-43.
10. Leitinger M, Beniczky S, Rohracher A, Gardella E, Kalss G, Qerama E, et al. Salzburg consensus criteria for non-convulsive status epilepticusapproach to clinical application. Epilepsy Behav. 2015;49:158-63.

11. Urbain P, Bertz H. Monitoring for compliance with a ketogenic diet: what is the best time of day to test for urinary ketosis? Nutr Metab. 2016;13:1-6.

12. Arya R, Peariso K, Gaínza-Lein M, Harvey J, Bergin A, Brenton JN, et al. Efficacy and safety of ketogenic diet for treatment of pediatric convulsive refractory status epilepticus. Epilepsy Res. 2018;144:1-6.

13. Kantanen A-M, Reinikainen M, Parviainen I, Ruokonen E, Ala-Peijari M, Bäcklund T, et al. Incidence and mortality of super-refractory status epilepticus in adults. Epilepsy Behav. 2015;49:131-4.

14. Brophy GM, Bell R, Claassen J, Alldredge B, Bleck TP, Glauser T, et al. Guidelines for the evaluation and management of status epilepticus. Neurocrit Care. 2012;17:3-23.

15. Tian F, Heighes PT, Allingstrup MJ, Doig GS. Early enteral nutrition provided within 24 hours of ICU admission. Crit Care Med. 2018;46:1049-56.

16. Gaspard N, Foreman BP, Alvarez V, Cabrera Kang C, Probasco JC, Jongeling AC, et al. New-onset refractory status epilepticus: etiology, clinical features, and outcome. Neurology. 2015;85:1604-13. 